\section{Brain Functional Mechanisms in Attentional Processing Following Modified Conflict Stroop Task}

\author{
Jalalvandi M. ${ }^{1 \oplus}$, ZahediNiya M.2*৫, Kargar J.2, Karimi S. \\ A. ${ }^{3,4}$, Sharini H. ${ }^{5,6}$, Goodarzi N.7
}

\begin{abstract}
Background: Cognitive control of brain regions can be determined by the tasks involving the cognitive control such as the color word Stroop task. Stroop task define the reduction in function in incongruent condition, which requires more attention and control of competitive responses.
\end{abstract}

Objective: The purpose of this study was to evaluate the activity of brain using the Modified Conflict Stroop Task in Military Personnel.

Material and Methods: In this applied experimental study, to specify the activity of different regions of brain in response to conflict Persian color-word Stroop task, 20 healthy persons participated in this study. To evaluate selective attention, the traditional color-word Stroop Task Model was modified, and the Stroop test was designed in high- and low-threat zones. We used functional magnetic resonance imaging (fMRI) to evaluate the brain activation during the Stroop task performance. The color-word Stroop task consists of incongruent, congruent, and neutral conditions, and the subjects were requested to carefully choose the correct answer.

Results: The mean response time was longer in incongruent condition ( $867.6 \pm 193.5 \mathrm{~ms})$ compared to congruent and neutral conditions. Analysis of neuroimaging data revealed that the brain conflict-related regions are activated by the Stroop interference. In incongruent trial, the superior frontal gyrus (SFG) and inferior frontal gyrus (IFG) showed the most active and stronger BOLD responses. In congruent trials, the activation in the brain was less and had difference compared with incongruent trials.

Conclusion: Our result offers that the frontal cortex and the anterior cingulate cortex are sensitive to different trials of Persian Stroop task. Using modified Stroop task, we determined the brain responses to the selective attention test.

Citation: Jalalvandi M, ZahediNiya M, Kargar J, Karimi SA, Sharini H, Goodarzi N. Brain Functional Mechanisms in Attentional Processing Following Modified Conflict Stroop Task. J Biomed Phys Eng. 2020;10(4):493-506. doi: 10.31661/jbpe.v0i0.2003-1084.

\section{Keywords}

Stroop Test; Functional MRI; Cognition; Attention; Dorsolateral Prefrontal Cortex

\section{Introduction}

he identification of the brain neural networks in the functioning performance has always been the subject of discussion in cogniL tive neuroscience [1]. Cognitive control point to procedures underpinning the capability to hold goal-directed behavior in the attendance of competing origin of information that extract different or conflicting
${ }^{1} \mathrm{MSc}$, Department of

Radiology, School of Medi-

cine, AJA University of

Medical Science, Tehran,

Iran

${ }^{2} \mathrm{MD}$, Department of Radi-

ology, School of Medicine,

AJA University of Medical

Science, Tehran, Iran

${ }^{3} \mathrm{PhD}$, Neurophysiology

Research Center, Hama-

dan University of Medical

Sciences, Hamadan, Iran

${ }^{4} \mathrm{PhD}$, Department of

Neuroscience, School of

Science and Advanced

Technologies in Medicine,

Hamadan University of

Medical Sciences, Hama-

dan, Iran

${ }^{5} \mathrm{PhD}$, Department of

Biomedical Engineer-

ing, School of Medicine,

Kermanshah University of

Medical Sciences (KUMS)

Kermanshah, Iran

${ }^{6} \mathrm{PhD}$, Health Research

Center, Kermanshah

University of Medical Sci-

ences (KUMS), Kerman-

shah, Iran

${ }^{7} \mathrm{PhD}$, Department of

clinical phycology, School

of Medicine, AJA Univer-

sity of Medical Science,

Tehran, Iran

*Corresponding author:

M. ZahediNiya

Department of Radiol-

ogy, School of Medicine,

AJA University of Medical

Sciences, Tehran, Iran

E-mail: drmzn45@gmail.

\section{com}

Received: 16 March 2020

Accepted: 25 April 2020 
responses. Also, cognitive processes are controlled by several brain networks including the ventrolateral (VLPFC) and dorsolateral (DLPFC) prefrontal cortex, the posterior parietal, anterior cingulate (ACC) and premotor cortices, the cerebellum and the basal ganglia. Each of these parts of brain displays a degree of specialization during different steps of cognitive control $[2,3]$. The (ACC) and (DLPFC) are involved in conflict processing [4]. In taskrelevant procedures, the VLPFC is involved in response performance [5].

Fast learning reactions a type of updating that is known as conflict conformity [4], in which a successful resolution of conflicting answer cues improvement of the effectiveness of resolving similar conflicts in the future [6]. One of the most important cognitive tasks is the Stroop Color or Word task, which is apparently related to the brain attention processing [7]. The Stroop task is one of the most important cognition tasks to find deficits in attentional control processes such as inhibition and switching [8]. Stroop task is surely one of, if not just the most studied task in psychology and remains at the foundation of studies into human selective attention and cognitive control $[9,10]$.

As a basic principle, the Stroop task shows that recognizing the ink-color of the words independent from the written color name (incongruent condition) is always more difficult than simply reading the names of color alone (congruent condition), which this event is called Stroop interference [11]. Stroop interference is specified by the slowed response to naming these incongruent words compared to congruent or neutral stimuli. Stroop facilitation measures the speed of response to naming congruent words and incongruent stimuli [12]. Therefore, it is assumed that some attentional and cognitive functions such as interference resolution, response inhibition, and an individual's response speed can be evaluated by Stroop task [13].

Nowadays, advanced brain mapping meth- ods have enabled us to understand the relationship between the activation of different brain regions and cognition. Therefore, we are able to study the different parts of human brain in a wide range of activities $[14,15]$. Functional Magnetic Resonance Imaging (fMRI) is one of the best neuroimaging methods, which has the ability to identify the activity of brain regions involved in different cognitive tasks $[16,17]$. In fMRI, the activation of different brain regions is measured by signal amplitude changes depending on blood-oxygenationlevel dependent (BOLD) contrast [18,19].

Studies conducted on the conflict monitoring theory usually highlighted the role of the dorsolateral prefrontal cortex (DLPFC) and dorsal anterior cingulate cortex (ACC) in conflict processing [4]. Studies performed using electrophysiological and neuroimaging techniques have shown the BOLD signal variations in both the ACC and DLPFC cortex [20-23]. However, these areas are not the only brain-active areas in conflict tasks, activation in the superior frontal gyrus (SFG), superior parietal lobe, and cerebellum that are recorded [24]. The current study aimed to evaluate the brain activation in correlation with behavioral response following Stroop Task.

Monitoring brain hemodynamic changes in response to selective attention stimuli and identifying different areas of the brain, play important roles in promoting and enhancing the focusing techniques by considering and following selective attention stimuli. People's attention and accuracy are closely linked to job performance, especially when one needs to perform accurate, error-free, and fast. Accordingly, this connection is much livelier for those who work in military units because the experiences belonged to numerous humans in wars throughout history have shown that one of the key factors in the success of military units in battlefields is the presence of staff with high mental readiness [25].

Researchers proposed a hypothetical that military personnel require low time for cogni- 
Brain Functional Mechanism in Attentional Processing

tive processing of external stimulus enhanced reaction precision rate compared to ordinary people. The present study was aimed to evaluate the brain activity in correlation with behavioral reaction following Stroop Task in military personnel. If this hypothesis is proven, it can be said that interventional tactics to increase the psychological wellbeing of military personnel can be performed to improve combat mission performance.

\section{Material and Methods}

This applied experimental study aimed to evaluate the brain activation in correlation with behavioral response following Stroop Task among military personnel. In this applied research, the fMRI data were analyzed using GLM to detect the activation of brain regions correlated to behavioral response following Stroop Task in military personnel. Before describing these methods, we should describe the appropriate model structure more particularly. In this study, we focused on several regions of the brain cortex related to selective attention.

\section{Participants}

20 healthy men (mean age $28.2 \pm 2.54$ years old) were chosen for this study, which was approved by the Aja University of Medical Sciences Human Ethics Committee. All subjects were right-handed and attentively screened for no history of neurological, psychiatric, and visual or substance abuse disorders. Participants were all native Persian speakers. A written informed agreement was taken from all participants. The protocol of the human study was approved by the Local Ethics Committee of AJAUMS (approval number: IR.AJAUMS. REC.1398.258).

\section{Stroop task}

Traditional patterns of Stroop task were performed to evaluate automated and controlled processing of attention stimuli, which require the tester to make little effort to respond to the test. In this study, to fully simulate the work environment, and also to more accurately assess the selective attention of military personnel, the patterns of Stroop Task were modified to simulate the work environment of military personnel. For this purpose, the color-word Stroop pattern was displayed in a simulated military environment context. As a result, military personnel need more attention and effort to choose the right answer. The modified pattern was randomly rendered to simulate the work environment of military personnel in color-word Stroop in two different contexts as follows: high- and low-threat zones. Regarding, low-threat zones display peace background images, and high-threat zones backgrounds display war situations, and people were asked to select the right answer in all 3 modes of incongruent, congruent, and neutral.

Participants executed the color-word Stroop task consist of incongruent, congruent, and neutral trials while in the MRI scanner. Also, the subjects were trained to deny the meaning of the printed word and just answer to ink color of the printed word. In congruent trial, the subjects observed a series of Persian color names mean '(blue)', '(green)', '(red)' and (yellow) were shown in the ink colors blue, green, red, and yellow respectively. In this trial, the need to attention was low because the color of the ink matched the meaning of the word written, so the response conflict was at least.

In incongruent trial, printed words were different from their ink colors, so that the participants were asked to check the color of ink independent from the written color name, which led to color name and color word interference (e.g., the word "Blue" was printed in Yellow ink).

This trial evoked conflict because answering corresponds to the printed word would lead to a wrong answer. As a result, the need to attention was high and subjects needed to prevent the primary response of reading the word and answering according to the ink color in which the word was printed (Figure 1).

In this study, we used 8 block Stroop tasks, 


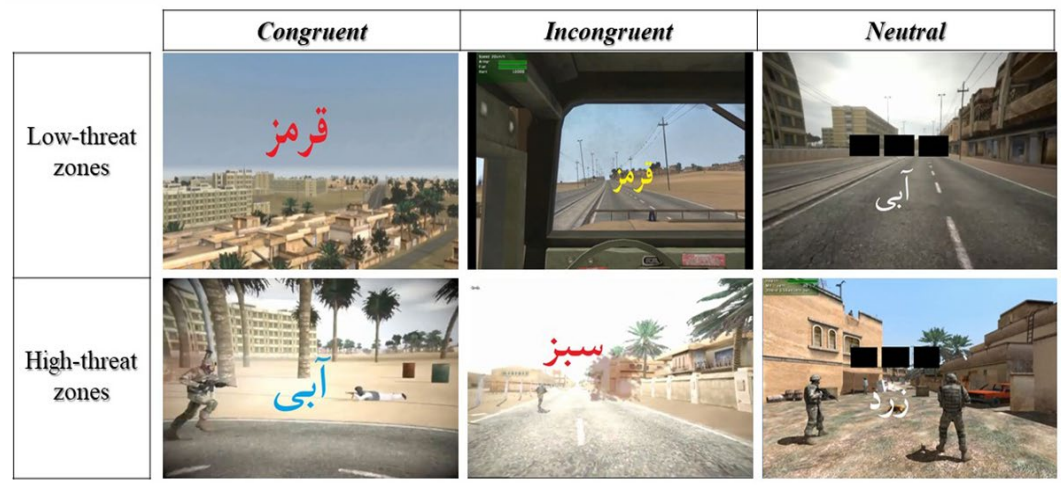

Figure 1: Samples of the different trials of Persian version of conflict Stroop Task. (a) Low- threat zones, (b) High-threat zones.

each block included 15 trials. A total of 120 trials (60 trials in low threat zones and 60 trials in high threat zones) randomly were showed to each volunteer (40 incongruent, 40 neutral, 40 congruent). The time interval between each block was 15 second. Before fMRI measurements, all volunteers performed both congruent and incongruent trial several times, for the reason of verifying their correct understanding of the conflict Stroop task paradigm by the test designers. The interstimulus interval between different trials was between 1.5-4 second, each trial was shown for $2 \mathrm{~s}$.

\section{Image acquisition}

The fMRI data were obtained using a 3Tesla Magnetic Resonance Imaging (Siemens, MAGNETOM Prisma) System with a standard 20 channel head coil. A high resolution T1-weighted image of brain was derived for all subjects consisting of 176 continuous slices with a MPRAGE sequence $(\mathrm{TR}=2300 \mathrm{~ms}$, TE $=2.97 \mathrm{~ms}$, field of view $=259 \mathrm{~mm}$, acquisition matrix $=256 \times 256$, voxel size $=1 \mathrm{~mm}^{3}$ ).

The EPI (echo planar imaging) sequence was executed with an echo time (TE) of $20 \mathrm{~ms}$ and a repetition time (TR) of $1500 \mathrm{~ms}$ and with $90^{\circ}$ flip angle. 30 continues slices $(3.2 \mathrm{~mm} \times 3.2$ $\mathrm{mm} \times 4 \mathrm{~mm}$ ) were collected.

\section{Behavioral analysis}

Response times (RT) for correct answers were calculated for each subject in all conditions. Response times were calculated as the time between the start of onset and key press (in milliseconds). All behavioral analyses were limited to accurate responses. To do Behavioral analysis, an ANOVA test was used at a significance level of $5 \%$.

\section{fMRI Data Analysis}

Functional collected data obtained from MRI Scanner were processed and then analyzed with the SPM12 toolbox (http://www.fil. ion.ucl.ac.uk/spm/software/spm12), running on MATLAB 2016a. Pre-processing steps included the following: Field map correction, co-registration of functional and anatomical scans, realignment, segmentation, normalization, and smoothing. In the first step, we used field map correction toolbox to accurate the signal changes because of non-uniformity in the magnetic field of MRI scanner. The purpose of realignment step was to remove movement artefact in fMRI data, in this step, all volumes of fMRI data were spatially realigned to the first volume of fMRI series. In the co-registration step, unwrapped mean fMRI echo planar images were co-registered to the anatomical image (T1 weighted images). In normalization step, co-registration images were normalized with the Montreal Neurological Institute (MNI) atlas, and in smoothing step, we used Gaussian kernel with a specified width. 
Brain activations were estimated by SPM12 toolbox. Analysis was executed at two levels as follows: first level and group level analyses. In the first level analysis, the maps of brain neural activation for each person were obtained using a linear multiple regression model with 3 event-related regressors (one for each condition containing: congruent, incongruent, and neutral). In the group analysis for calculation of the similarity and dissimilarity among the subject's data, we could investigate brain activity patterns related to different conditions. We used $p=0.001$ to limit the number of false positive voxels.

\section{General Linear Model (GLM) and fMRI Data}

The General Linear Model (GLM) is a powerful method for fMRI data analysis, and it was used to compute the amount of activity variation in different conditions.

GLM has the ability to integrate multiple qualitative and quantitative independent factors and can be expressed using the Equation form containing: Predictors, Parameters, and Error [24].

$$
\mathbf{Y}=\mathbf{X} \cdot \boldsymbol{\beta}+\boldsymbol{\varepsilon}
$$

Observed $=$ Predictors $\times$ Parameters + Error y: Voxel time series
$\mathrm{X}$ : Predictors
B: Beta values
\&: residuals

In this equation, $\mathrm{X}$ demonstrates the design matrix containing the predictor time series. The beta values indicate differences in brain activity of different regions relative to the modeled baseline signal [26].

\section{Results}

\section{(a) Behavioral Results}

The mean RT for the neutral condition was $(777.6 \pm 154.1 \mathrm{~ms})$, and for the congruent condition it was $(734.5 \pm 121.8 \mathrm{~ms})$. The mean response times in neutral and congruent conditions were shorter than the incongruent condition ( $867.6 \pm 193.5 \mathrm{~ms})$. In incongruent conditions, the average errors were $3 \%$, and in congruent conditions the average errors were $2 \%$ (Figure 2).

\section{(b) fMRI Data Analysis Result}

To extract different brain regions associated with the response incongruency stimulus,

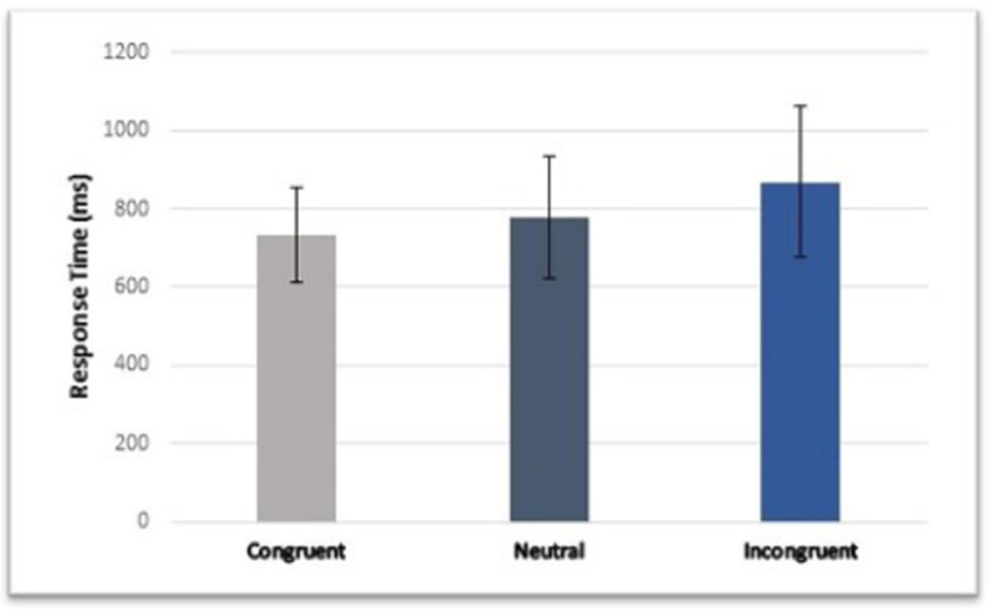

Figure 2: Behavioral response time during the conflict Stroop task. Results of data analysis show that response times (RTs) for precise incongruent conditions were slower compared with neutral conditions, while RTs for congruent conditions were faster compared with neutral conditions. 
a random effect analysis was performed and identified the brain regions that respond to the incongruent and congruent conditions compared with neutral conditions. This contrast was executed to extract different brain parts that are selectively responsive to incongruent stimulus.

Several different areas and different brain regions related to task special reactions during incongruent trials compared with neutral trials were activated (Figure 3 and Table 1). Superior frontal gyrus $\left(\mathrm{N}_{\mathrm{vox}}=232\right.$, $\mathrm{t} \_$value $\left.=5.6058\right)$, one of the brain regions, shows a large cluster of activated voxels that had a powerful BOLD response during incongruent conditions. Our data analysis of incongruent conditions indicated that other different brain areas were also activated such as bilateral middle frontal gyrus(MFG), right cingulate gyrus, bilateral inferior Frontal gyrus(IFG), and other differ- ent brain parts indicated in Table 1 .

A whole brain group analysis in congruent trials compared to neutral trials indicated that different parts of brain were activated. Our extracted brain activation maps of congruent trials show the middle temporal gyrus $\left(\mathrm{N}_{\mathrm{vox}}\right.$ $=71, \mathrm{t}$ _value $=-4.5693$ ) had a large number of activated voxels compared to other brain regions. In addition to the middle temporal gyrus(MTG), other areas in the brain also respond to the congruent trials such as bilateral middle frontal gyrus(MFG), superior temporal gyrus, and other different brain parts indicated in Figure 4 and Table 2.

\section{Comparison of brain activation patterns in incongruent and con- gruent trials}

fMRI data analysis results show that activation maps were wider and more areas of the

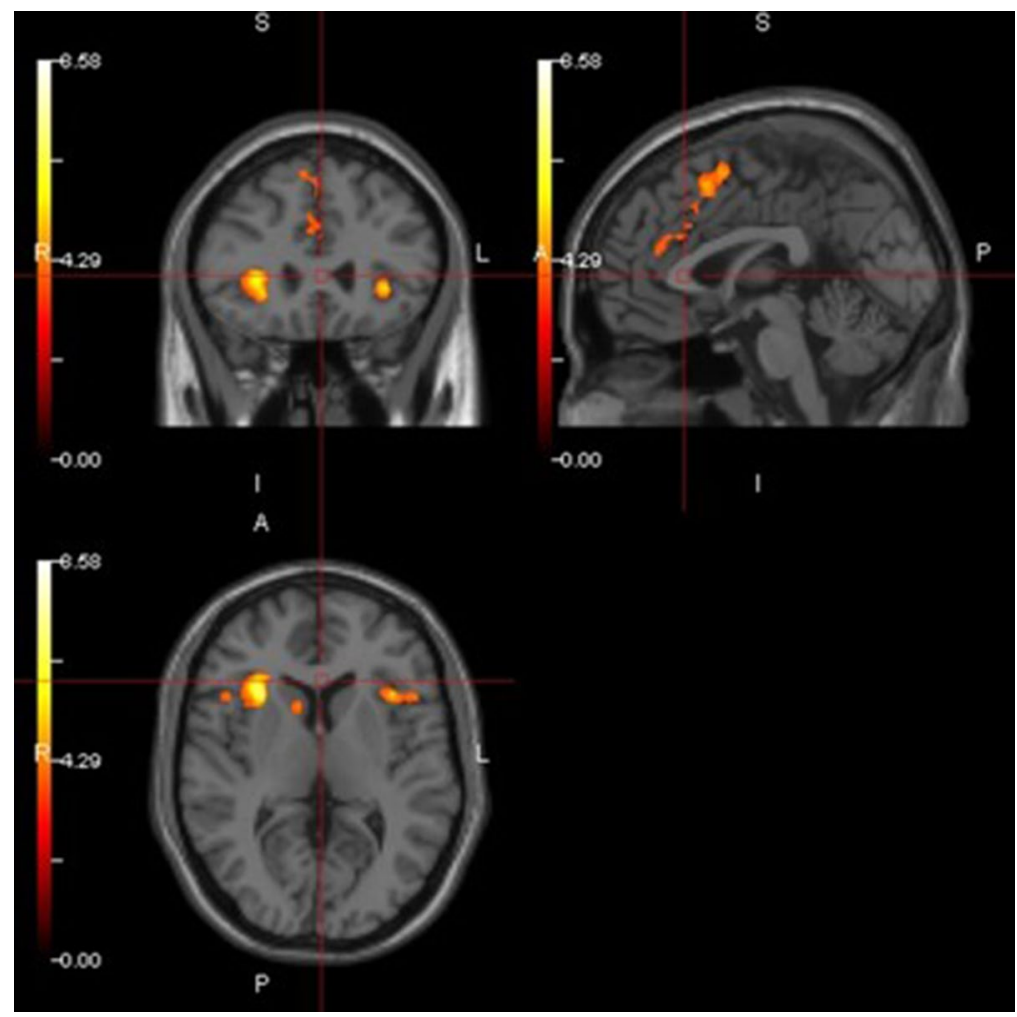

Figure 3: Brain activation maps for conflict Stroop task obtained from SPM 12. Brain activation maps displaying voxels with different BOLD responses to cue incongruent trials (incongruent vs. neutral trials) in distinct regions of brain. 
Brain Functional Mechanism in Attentional Processing

Table 1: Brain cortical activations in incongruent trial compared with neutral trials (incongruent $>$ neutral).

\begin{tabular}{lcccc}
\multicolumn{1}{c}{ ROI } & BA & $\mathbf{N}_{\text {vox }}$ & MNI Coordinates $\mathbf{( x , y , z )}$ & Peak t \\
\hline R_superior frontal gyrus & 11 & 232 & 92062 & 5.6058 \\
\hline L_middle frontal gyrus & 46 & 132 & -452026 & 7.7673 \\
\hline R_middle frontal gyrus & 9 & 107 & 451729 & 5.6251 \\
\hline Precuneus & 7 & 28 & $-3-7044$ & 3.6713 \\
\hline R_cingulate gyrus & 6,32 & 26 & $3-1926$ & 5.408 \\
\hline R_middle temporal gyrus & 21 & 37 & $48-25-7$ & 4.1446 \\
\hline R_superior temporal gyrus & 22 & 56 & $57-4920$ & 4.8018 \\
\hline R_inferior parietal lobule & 39 & 109 & $36-4338$ & 5.1916 \\
\hline L_inferior parietal lobule & 39 & 113 & $-33-4938$ & 4.9788 \\
\hline R_inferior frontal gyrus & 45 & 221 & 33235 & 8.2679 \\
\hline L_inferior frontal gyrus & 47 & 191 & $-3326-1$ & 5.9711 \\
\hline L_supramarginal gyrus & 40 & 58 & $-60-4932$ & 4.9704
\end{tabular}

Note: right (R) or left (L) brain hemisphere; BA: Brodmann area; voxels: number of activated voxels in a cluster, only clusters 20 voxels or greater; MNI Coordinates (x, y, z): X, Y, Z in MNI Atlas; Peak t values from a t-test of the peak voxel (displaying the greatest statistical difference within a cluster).

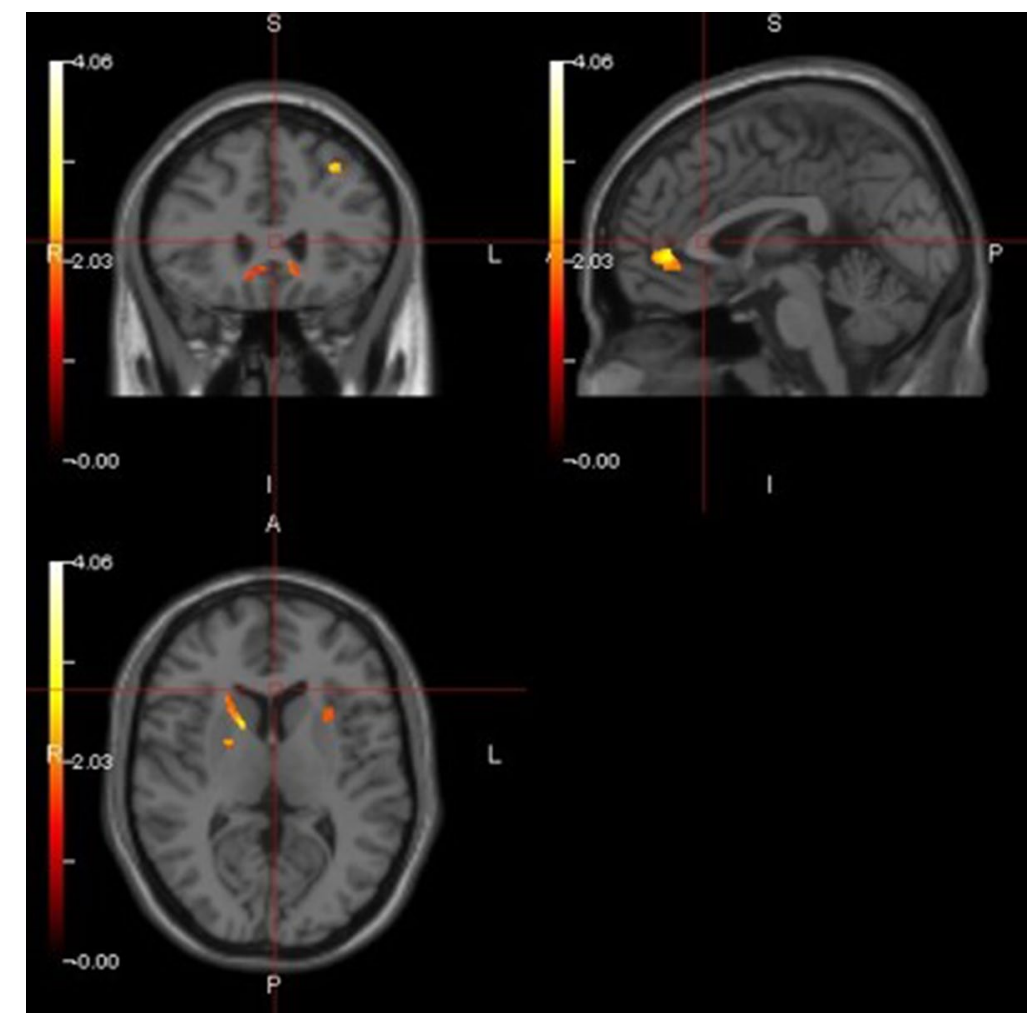

Figure 4: Brain activation maps for conflict Stroop task obtained from SPM 12. Brain activation maps displaying voxels with different BOLD responses to cue congruent trials (congruent vs. neutral trials) in distinct regions of brain. 
Table 2: Brain cortical activations in incongruent trial compared with neutral trials (congruent $>$ neutral).

\begin{tabular}{lcccc}
\multicolumn{1}{c}{ ROI } & BA & N $_{\text {vox }}$ & MNI Coordinates $(\mathbf{x}, \mathbf{y}, \mathbf{z})$ & Peak t \\
\hline L_middle frontal gyrus & 46 & 58 & -301738 & 3.8547 \\
\hline R_middle frontal gyrus & 46 & 44 & 30844 & 4.0522 \\
\hline R_superior frontal gyrus & 11 & 36 & 273253 & 3.775 \\
\hline L_superior temporal gyrus & 22 & 32 & $-63-3111$ & -4.5853 \\
\hline R_parahippocampa gyrus & 36 & 59 & $36-28-19$ & -4.2487 \\
\hline R_middle temporal gyrus & 21 & 71 & $54-37-16$ & 4.5693 \\
\hline L_middle temporal gyrus & 21 & 47 & $-36-7023$ & -3.6633
\end{tabular}

Note: right (R) or left (L) brain hemisphere; BA: Brodmann area; voxels: number of activated voxels in a cluster, only clusters 20 voxels or greater; MNI Coordinates (x, y, z): X, Y, Z in MNI Atlas; Peak t values from a t-test of the peak voxel (displaying the greatest statistical difference within a cluster).

brain were activated in incongruent conditions compared to congruent conditions in incongruent trials. By comparing the incongruent conditions with the congruent conditions, in the inferior frontal gyrus $\left(\mathrm{N}_{\mathrm{vox}}=91\right.$, $\mathrm{t}$ _value $=7.9019$ ) more pixels were activated, and the activations maps were completely separated and differed. In incongruent trials, more brain regions were activated compared to the congruent trials such as bilateral middle frontal gyrus, medial frontal gyrus, and other different brain parts indicated in Figure 5 and Table 3. Figure 6 shows $3 \mathrm{D}$ patterns of the activation maps in incongruent and congruent conditions.

\section{Discussion}

In the present study, we used a cognition block design fMRI task to recognize different parts of the brain involved in the stimulus incongruency. In this research, we used fMRI imaging device to indicate the brain responses to the conflict Stroop task. Statistical parametric Mapping (SPM) analysis demonstrated that concentration changes in BOLD responses of differential brain regions were extracted during the conflict Stroop interference. The results of this study demonstrate different activation maps in several brain regions.

The main goal of the current study was to evaluate the differences in functional processes underlying the incongruent and the congruent effects. We found that various brain areas such as the superior frontal gyrus and inferior frontal gyrus were powerfully activated by the incongruent test. We found that the prefrontal cortex and particularly the cingulate gyrus, supramarginal gyrus, and inferior frontal gyrus were more sensitive to the incongruent effect, and these regions were not activated in congruent trials.

Selective attention mention to the ability to directly process of information changes when exposed to particular objects, spacial locations, and executive functions, which can be usually defined as a process that guides thoughts and actions. A bunch of stimulus-response executive functions was used to measure selective attention. These executable functions include Stroop tests. The Stroop test is a good test for assessing the attention because it measures the selective focus, accuracy, and attention in response to the stimulus in a short time.

On the other hand, identifying the ability to distinguish between high significant levels and low significant levels of tasks is essential to identify and prevent inefficiencies during for example operations (including military operations), piloting, driving. One of the most important research applications in that field is 


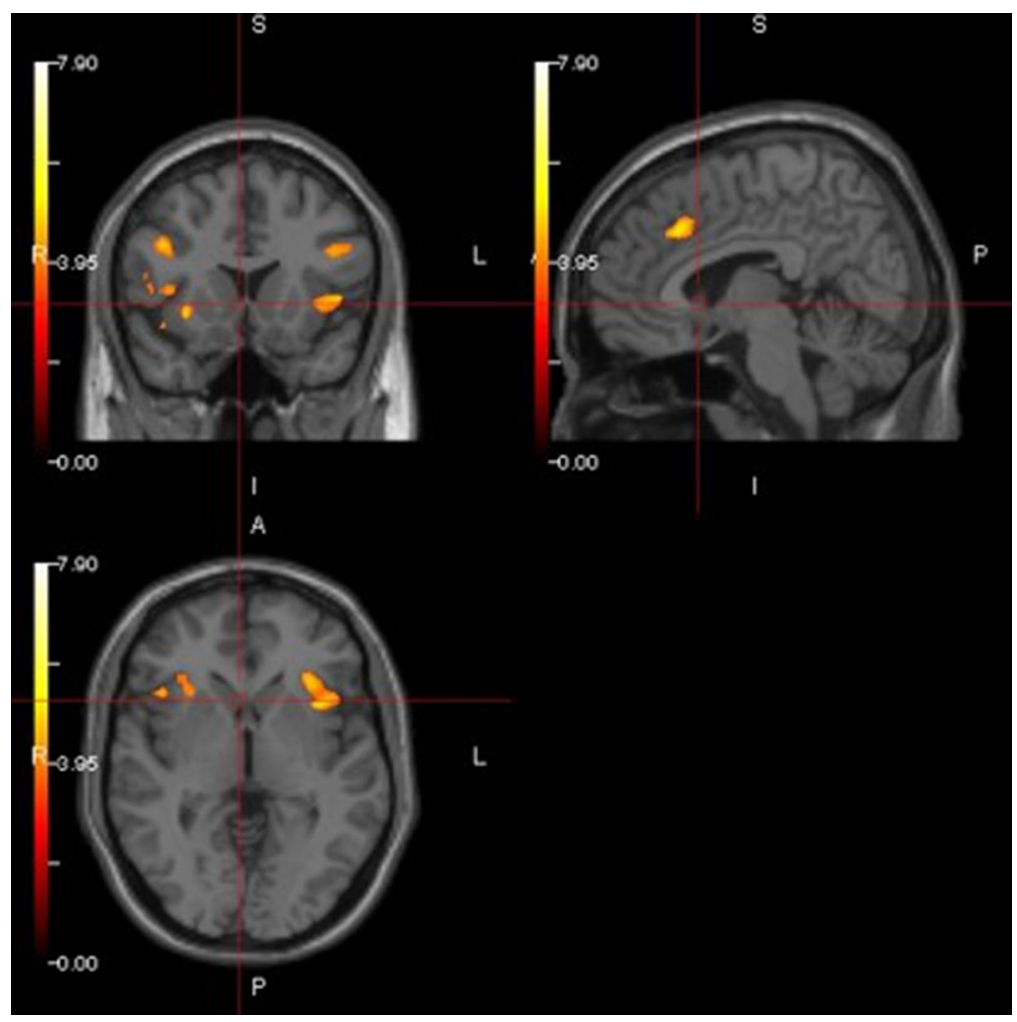

Figure 5: Brain activation maps for conflict Stroop task obtained from SPM 12. Differential brain activation maps displaying voxels with BOLD responses to cue incongruent trials compared with congruent trials in distinct regions of brain. This activation map shows pure brain activity obtained from the differences in incongruent conditions than to the congruent conditions.

Table 3: Brain cortical activations in incongruent trial compared with neutral trials (incongruent $>$ congruent).

\begin{tabular}{lcccc}
\multicolumn{1}{c}{ ROI } & BA & N $_{\text {vox }}$ & MNI Coordinates $\mathbf{( x , y , ~ z ) ~}$ & Peak t \\
\hline R_inferior frontal gyrus & 45 & 91 & 30238 & 7.9019 \\
\hline L_inferior frontal gyrus & 47 & 59 & -45172 & 5.7292 \\
\hline R_medial frontal gyrus & 8 & 28 & $647-13$ & -5.8217 \\
\hline R_superior temporal gyrus & 22 & 34 & $63-4617$ & 5.4732 \\
\hline L_middle frontal gyrus & 46 & 46 & -39241 & 4.7572 \\
\hline R_middle frontal gyrus & 9 & 31 & 452026 & 6.0435
\end{tabular}

Note: right (R) or left (L) brain hemisphere; BA: Brodmann area; voxels: number of activated voxels in a cluster, only clusters 20 voxels or greater; MNI Coordinates (x, y, z): X, Y, Z in MNI Atlas; Peak t values from a t-test of the peak voxel (displaying the greatest statistical difference within a cluster).

in the aviation industry and in the safety of autopilot flights, which has also been addressed by the US Department of Defense. This is also more important in astronauts [27].

Emotional control and attention retention in specific situations, especially in the opera- 

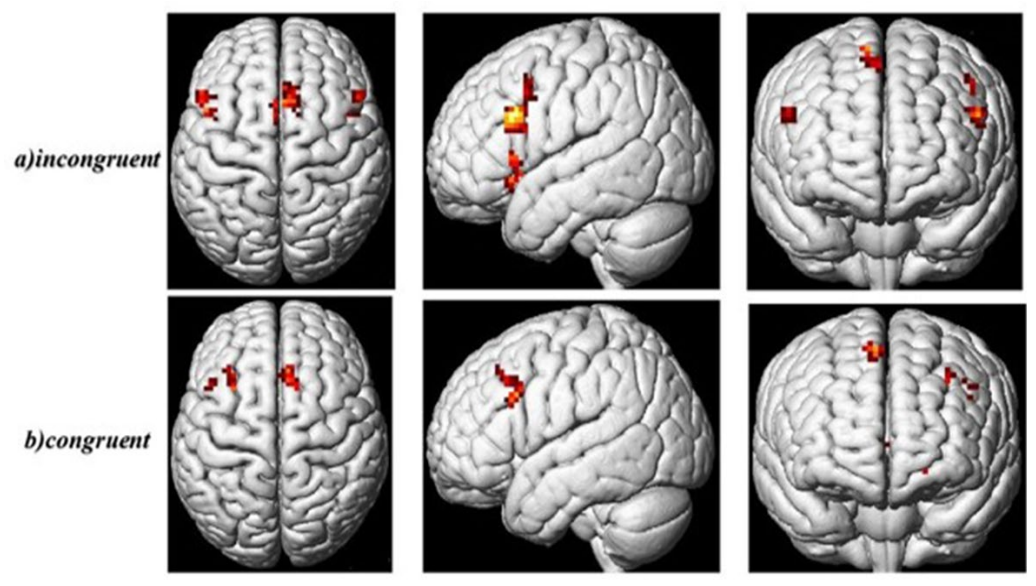

Figure 6: 3D patterns showing the activation maps in Incongruent and congruent conditions. a) Brain activations patterns in incongruent trials. b) Brain activations patterns in congruent trials. These 3D patterns show the different brain activations maps in two conditions (incongruent and congruent).

tional and military forces, and those who require a high level of attention to perform their duties are important subjects in military and psychological studies, as Perth et al. found in their study sponsored by the US Army. They examined selective attention among different people [28]. In general, the occurrence of neural activity in the brain is associated with different and complex physiological activities, which some of these processes cause alter physical parameters.

Overall, it can be stated that by measuring some of the secondary changes caused by neural activity, it is possible to infer the activity and its location. Studying and determining the patterns of brain activities can be an effective tool for assessing the cognitive status, and in particular, identifying the high and low levels of task participation. The use of stimulus-response tests (such as Stroop) allows for the assessment of selective attention (in which one must give the stimulus a correct and correct response), and with these tests, it is possible to examine the changes in neural activity. fMRI provides many details on physiological and functional properties, and imaging using functional magnetic resonance imaging provides a unique opportunity to examine concentration changes within the small vascular space with high spatial resolution. fMRI has been widely applied for studying the brain activity because of its high spatial resolution, noninvasiveness, and high signal-to-noise ratio, and also this method reflects brain activity imaging. Also, fMRI allows for depth imaging of brain tissue, which has been proven as a useful tool for clinic and out-of-clinic applications. Nowadays, Functional magnetic resonance imaging is widely used to evaluate visual, auditory, motor, and cognitive stimuli [29].

Our behavioral data analysis displayed that response times (RTs) were slower in incongruent trials compared to neutral and congruent trials, which indicate the expected conflict Stroop effect. This analysis indicated that prolonged activity for incongruent trials created a conflict in choosing the right answer, compared to congruent stimuli. The BOLD activation maps for different Stroop conditions demonstrated the maximum activity in the prefrontal cortex, which is in accordance with previous studies [30,31].

The different brain regions were activated by incongruent and congruent trials, which demonstrated that these two kinds of trials have various underlying mechanisms [32]. In 
incongruent trials, more regions of the brain were activated compared to congruent trials. Many of these areas were identified in previous studies as those involved in incongruency processes in the Stroop task [33]. The right superior frontal gyrus (BA11) and the right inferior frontal gyrus (BA45) are prefrontal brain regions that were generally activated by the incongruent trials (see Table 1). Our data results demonstrate that the prefrontal region plays a significant role in cognitive processes during both congruent and incongruent trials. Results of previous studies and the current study consistently showed that the prefrontal cortex is more necessary for cognitive control, and the brain prefrontal cortical parts may be involved in conflict processes [34-36].

The focal cortical brain regions associated with the Stroop Color-Word test are not fully identified. Stroop Color-Word test needs the ability to actively inhibit an overlearned reaction in favor of a more voluntary response and it is extensively used as an indicator of attention and executive control. In our work, we showed that Stroop Color-Word test produces response latency, and we found that this paradigm activates different prefrontal areas, including the superior frontal gyrus, the left inferior frontal gyrus (BA 9) and bilateral middle frontal gyrus (BA 9).

Imaging studies provided the first evidence for anterior cingulate cortex (ACC) contribution during the Stroop interference subtest. Pardo et al. using positron emission tomography (PET), stated the elevation of regional cerebral blood flow (rCBF) of the ACC during the Stroop attentional conflict paradigm [37]. Peterson et al. used fMRI to examine Stroop performance in healthy subjects during incongruent and congruent trials with procedures nearly similar to our work. They found some evidence for cingulate subregions contribution to this task [38]. Moreover, imaging studies indicated the increased signal intensity within the prefrontal regions as well as ACC in answer to spatial working memory challenge paradigms
[39]. Results from our work show that significant variations in signal intensity are present in the bilateral middle frontal gyrus, superior frontal gyrus, and the left inferior frontal gyrus of subjects when the interference trials of the Stroop Color Word Test are compared to baseline states. The results of our study show further evidence that, in addition to the cingulate cortex, frontal regions also play an essential role in this task. Newly, studies on the visual word recognition proposed that there are some distinctions between alphabetic systems (e.g., English) and ideographic systems (e.g., Chinese and maybe Persian) [40].

By comparing these findings with previous findings, it seems that Persian (Farsi) Stroop interference has a consistent time courses and had a topographical map over frontocentral scalp parts. During performing the Stroop test of color naming of Persian characters, despite an intentional effort on the subject's part to attend to the ink color of words; the information of color and meaning will inevitably bring about conflict in the incongruent trial, due to the automation of the processing of word meaning. Then, the activity of the ACC or FC is required to control the irrelative information inference (semantic information) and make the right judgment in terms of the information of color.

Superior frontal gyrus, one of the brain regions, showed a large cluster of activated voxels that had powerful BOLD responses during incongruent conditions. Superior frontal gyrus is specified by a dense layer II that is understandably separated from layer III. Layer III consists of medium and large pyramidal cells, as well as very large and dark stained pyramidal neurons that occupy the lower part of this layer [41]. The superior frontal gyrus (SFG) is thought to contribute to higher cognitive functions and especially to working memory [42]. In the frontal and prefrontal cortex, neurons responsive to visual, somatosensory, auditory, and olfactory stimuli have been shown. However, combined behavioral and neurophysi- 
ological researches have shown that responses can considerably vary with the behavioral importance of the stimulus [43].

Complex stimuli have a longer response time than simple stimuli because they are more difficult to initiate in response, which can increase the effectiveness of the psychological inactivity period. Accordingly, it is important in military missions this time, as well as the incongruous state of the Stroop effect increases the complexity of the military stimulus analysis process, because the relevant stimuli are identified among the factors that cause distraction. In this study, we attempted to evaluate the control attention using a task that is close to the military environment and Iranian conditions. In fact, this study is a creative study using Persian and condition-compatible Stroop test, and given these conditions, it is likely that the results will be more realistic than ordinary Task and more realistic evaluation will be achieved, because additional Task in a virtual environment may require additional cognitive needs than traditional versions of neuropsychological evaluations.

In this study, we examined the results of activity analyzes of different brain regions to assess the effects of low and high threat zones on people's attention. But no significant difference was found between the two conditions in the military subjects. This result was probably because most of the military's attention was focused on Stroop's Task and little attention was paid to environmental conditions. The results indicate the need for further studies using more advanced tasks such as virtual reality tasks.

\section{Conclusion}

In the present study, we attempted to determine the brain activity and the brain cortical regions differentially of military officers that were activated by the Persian Stroop test and the current study is the developmental fMRI study of the color-word Stroop test. Specifically, we examined this hypothesis that differ- ent mechanisms involved in the different interference types of Stroop task.

Our results showed that, specifically, activity in the frontal cortex and the anterior cingulate increased during Stroop task. The activation patterns were different in congruent and incongruent conditions, and these results offer that congruent and incongruent trials can be attributed to different behavioral and neural mechanisms. The current findings show that response time for cognitive processing of external information (incongruent conditions), was longer than neutral and congruent conditions, which indicates that the brain's processing speed is slower in complex states than in simpler states. Based on the current study results, we proposed the application of the Persian color-word Stroop task for examining individual differences in cognitive control among military officers. This research provided the fMRI entry point for assessing military readiness, including attention. It is recommended that such studies should be performed in other psychological tasks, including Flanker.

\section{Acknowledgment}

This work was supported in part by the Research Deputy of AJA University of Medical Sciences, project grant No. of (97000864). The authors wish to thank AJA University of Medical Sciences for the financial and instrumental support of this research.

\section{Conflict of Interest}

None

\section{References}

1. Schacter DL. The seven sins of memory: insights from psychology and cognitive neuroscience. American psychologist. 1999;54(3):182. doi: 10.1037//0003-066x.54.3.182. PubMed PMID: 10199218 .

2. Aron AR, Durston S, Eagle DM, Logan GD, Stinear $\mathrm{CM}$, Stuphorn V. Converging evidence for a frontobasal-ganglia network for inhibitory control of action and cognition. J Neurosci. 2007;27(44):118604. doi: 10.1523/JNEUROSCI.3644-07.2007. PMID: 
17978025. PubMed PMCID: PMC6673355.

3. Nee DE, Wager TD, Jonides J. Interference resolution: insights from a meta-analysis of neuroimaging tasks. Cogn Affect Behav Neurosci. 2007;7(1):1-17. doi: 10.3758/cabn.7.1.1. PubMed PMID: 17598730.

4. Botvinick MM, Braver TS, Barch DM, Carter CS, Cohen JD. Conflict monitoring and cognitive control. Psychological review. 2001;108(3):624-52. doi: 10.1037/0033-295X.108.3.624.

5. Laird AR, McMillan KM, Lancaster JL, et al. A comparison of label-based review and ALE metaanalysis in the Stroop task. Human brain mapping. 2005;25(1):6-21. doi: 10.1002/hbm.20129. PubMed PMID: 15846823. PubMed PMCID: PMC6871676.

6. Egner T. Congruency sequence effects and cognitive control. Cogn Affect Behav Neurosci. 2007;7(4):380-90. doi: 10.3758/cabn.7.4.380. PubMed PMID: 18189011.

7. Stroop JR. Studies of interference in serial verbal reactions. Journal of experimental psychology. 1935;18(6):643-62. doi.org/10.1037/h0054651.

8. Lezak MD, Howieson DB, Loring DW, Fischer JS. Neuropsychological assessment. USA: Oxford University Press; 2004.

9. Banich MT, Milham MP, Jacobson BL, et al. Attentional selection and the processing of task-irrelevant information: insights from fMRI examinations of the Stroop task. Prog Brain Res. 2001;134:45970. doi: 10.1016/s0079-6123(01)34030-x. PubMed PMID: 11702561.

10. Miller EK, Cohen JD. An integrative theory of prefrontal cortex function. Annu Rev Neurosci. 2001;24:167-202. doi: 10.1146/annurev.neuro.24.1.167. PubMed PMID: 11283309.

11. Williams JM, Mathews A, MacLeod C. The emotional Stroop task and psychopathology. Psychol Bull. 1996;120(1):3-24. doi: 10.1037/00332909.120.1.3. PubMed PMID: 8711015.

12. Carter CS, Mintun M, Cohen JD. Interference and facilitation effects during selective attention: an H2150 PET study of Stroop task performance. Neuroimage. 1995;2(4):264-72. doi: 10.1006/ nimg.1995.1034. PubMed PMID: 9343611.

13. Kane MJ, Engle RW. Working-memory capacity and the control of attention: the contributions of goal neglect, response competition, and task set to Stroop interference. J Exp Psychol Gen. 2003;132(1):47-70. doi: 10.1037/00963445.132.1.47. PubMed PMID: 12656297.

14. Han K, Kim IY, Kim JJ. Assessment of cognitive flexibility in real life using virtual reality: a comparison of healthy individuals and schizophrenia patients. Comput Biol Med. 2012;42(8):841-7. doi: 10.1016/j.compbiomed.2012.06.007. PubMed PMID: 22770745.

15. Sharini H, Riyahi Alam N, Jalalvandi M, et al. Assessment of Motor Cortex in Active, Passive and Imagery Wrist Movement Using Functional MRI. J Biomed Phys Eng. 2020.

16. Rajapakse JC, Piyaratna J. Bayesian approach to segmentation of statistical parametric maps. IEEE Transactions on biomedical engineering. 2001;48(10):1186-94. doi: 10.1109/10.951522.

17. Wang Y, Rajapakse JC. Contextual modeling of functional MR images with conditional random fields. IEEE transactions on medical imaging. 2006;25(6):804-12. doi: 10.1109/ TMI.2006.875426.

18. Goebel R, Roebroeck A, Kim DS, Formisano E. Investigating directed cortical interactions in time-resolved fMRI data using vector autoregressive modeling and Granger causality mapping. Magn Reson Imaging. 2003;21(10):1251-61. doi: 10.1016/j. mri.2003.08.026. PubMed PMID: 14725933.

19. Zheng X, Rajapakse JC. Learning functional structure from fMR images. Neuroimage. 2006;31(4):1601-13. doi: 10.1016/j.neuroimage.2006.01.031. PMID: 16540348.

20. Sheth SA, Abuelem T, Gale JT, Eskandar EN. Basal ganglia neurons dynamically facilitate exploration during associative learning. J Neurosci. 2011;31(13):4878-85. doi: 10.1523/JNEUROSCI.3658-10.2011. PubMed PMID: 21451026. PubMed PMCID: PMC3486636.

21. Sheth SA, Mian MK, Patel SR, Asaad WF, et al. Human dorsal anterior cingulate cortex neurons mediate ongoing behavioural adaptation. $\mathrm{Na}$ ture. 2012;488(7410):218-21. doi: 10.1038/nature11239. PubMed PMID: 22722841. PubMed PMCID: PMC3416924.

22. Kim C, Chung C, Kim J. Task-dependent response conflict monitoring and cognitive control in anterior cingulate and dorsolateral prefrontal cortices. Brain Res. 2013;1537:216-23. doi: 10.1016/j. brainres.2013.08.055. PubMed PMID: 24012877.

23. Kim C, Johnson NF, Gold BT. Conflict adaptation in prefrontal cortex: now you see it, now you don't. Cortex. 2014;50:76-85. doi: 10.1016/j. cortex.2013.08.011. PubMed PMID: 24074459. PubMed PMCID: PMC3872513.

24. Casey BJ, Thomas KM, Welsh TF, et al. Dissociation of response conflict, attentional selection, and expectancy with functional magnetic resonance imag- 
ing. Proc Natl Acad Sci U S A. 2000;97(15):872833. doi: 10.1073/pnas.97.15.8728. PubMed PMID: 10900023. PubMed PMCID: PMC27016.

25. Jalalvandi M, Riahi Alam N, Sharini H, Hashemi H, Kohzad S. Optical Imaging of the Motor Cortex in the Brain in Order to Determine the Direction of the Hand Movements Using Functional Near-Infrared Spectroscopy (fNIRS). Iranian Journal of Medical Physics. 2018;15(Special Issue-12th. Iranian Congress of Medical Physics):152. doi:10.22038/ ijmp.2018.12653.

26. Jalalvandi M, Riahi Alam N, Sharini H. Optical Imaging of Brain Motor Cortex Activation During Wrist Movement Using Functional Near-Infrared Spectroscopy (fNIRS). Arch Neurosci. 2019. doi: 10.5812/ans.90089.

27. Harrivel AR, Weissman DH, Noll DC, Peltier SJ. Monitoring attentional state with fNIRS. Front Hum Neurosci. 2013;7:861. doi: 10.3389/ fnhum.2013.00861. PubMed PMID: 24379771. PubMed PMCID: PMC3861695.

28. Pratt N, Willoughby A, Swick D. Effects of working memory load on visual selective attention: behavioral and electrophysiological evidence. Front Hum Neurosci. 2011;5:57. doi: 10.3389/ fnhum.2011.00057. PubMed PMID: 21716633. PubMed PMCID: PMC3115462.

29. Adorni R, Gatti A, Brugnera A, Sakatani K, Compare A. Could fNIRS promote neuroscience approach in clinical psychology? Front Psychol. 2016;7:456 doi: 10.3389/fpsyg.2016.00456. PubMed PMID: 27065924. PubMed PMCID: PMC4811970.

30. Prakash RS, Erickson KI, Colcombe SJ, et al. Age-related differences in the involvement of the prefrontal cortex in attentional control. Brain Cogn. 2009;71(3):328-35. doi: 10.1016/j. bandc.2009.07.005. PubMed PMID: 19699019. PubMed PMCID: PMC2783271.

31. Peterson BS, Kane MJ, Alexander GM, et al. An event-related functional MRI study comparing interference effects in the Simon and Stroop tasks. Brain Res Cogn Brain Res. 2002;13(3):427-40. doi: 10.1016/s0926-6410(02)00054-x. PubMed PMID: 11919006.

32. Song Y, Hakoda Y. An fMRI study of the functional mechanisms of Stroop/reverse-Stroop effects. Behav Brain Res. 2015;290:187-96. doi: 10.1016/j. bbr.2015.04.047. PubMed PMID: 25952963.

33. Banich MT, Milham MP, Atchley R, Cohen NJ, et al. fMRI studies of Stroop tasks reveal unique roles of anterior and posterior brain systems in attentional selection. J Cogn Neurosci. 2000;12(6):988-1000. doi: $\quad 10.1162 / 08989290051137521$. PubMed
PMID: 11177419.

34. MacDonald AW, Cohen JD, Stenger VA, Carter CS. Dissociating the role of the dorsolateral prefrontal and anterior cingulate cortex in cognitive control. Science. 2000;288(5472):1835-8. doi: 10.1126/science.288.5472.1835. PubMed PMID: 10846167.

35. Derrfuss J, Brass M, Neumann J, Von Cramon DY. Involvement of the inferior frontal junction in cognitive control: Meta-analyses of switching and Stroop studies. Hum Brain Mapp. 2005;25(1):2234. doi: 10.1002/hbm.20127. PubMed PMID: 15846824. PubMed PMCID: PMC6871679.

36. Brass M, Ruge $H$, Meiran N, Rubin 0, et al. When the same response has different meanings: recoding the response meaning in the lateral prefrontal cortex. Neuroimage. 2003;20(2):1026-31. doi: 10.1016/S1053-8119(03)00357-4. PubMed PMID: 14568472.

37. Pardo JV, Pardo PJ, Janer KW, Raichle ME. The anterior cingulate cortex mediates processing selection in the Stroop attentional conflict paradigm. Proc Natl Acad Sci U S A. 1990; 87(1): 256-259. doi: 10.1073/pnas.87.1.256. PubMed PMID: 2296583. PubMed PMCID: PMC53241.

38. Peterson BS, Skudlarski P, Gatenby JC, Zhang $\mathrm{H}$, et al. An fMRI Study of Stroop Word-Color Interference: Evidence for Cingulate Subregions Subserving Multiple Distributed Attentional Systems. Biol Psychiatry. 1999;45(10):1237-58. doi: 10.1016/s0006-3223(99)00056-6. PubMed PMID: 10349031.

39. Jonides J, Smith EE, Koeppe RA, Awh E, et al. Spatial working memory in humans as revealed by PET. Nature. 1993;363(6430):623-5. doi: 10.1038/363623a0. PubMed PMID: 8510752.

40. Qiu J, Luo Y, Wang Q, Zhang F, Zhang Q. Brain mechanism of Stroop interference effect in Chinese characters. Brain Research. Brain Res. 2006;1072(1):186-93. doi: 10.1016/j. brainres.2005.12.029. PubMed PMID: 16443198.

41. Mai JK, Paxinos G. The human nervous system. Academic press; 2011.

42. Boisgueheneuc FD, Levy R, Volle E, et al. Functions of the left superior frontal gyrus in humans: a lesion study. Brain. 2006;129(12):3315-28. doi: 10.1093/brain/awl244. PubMed PMID: 16984899.

43. Yousef Pour M, Masjoodi S, Fooladi M, et al. Identification of the Cognitive Interference Effect Related to Stroop stimulation: using Dynamic Causal Modeling of Effective Connectivity in Functional Near-Infrared Spectroscopy (fNIRS). J Biomed Phys Eng. 2020. 